\title{
Visual and CCD astrolabe observations of the solar radius
}

\author{
F. Noël` \\ Departamento de Astronomía, Universidad de Chile, Casilla 36-D, Santiago, Chile \\ Received 13 February 2001 / Accepted 2 May 2001

\begin{abstract}
Here we discuss and compare two sets of solar semidiameter measurements made with Danjon astrolabes during the period 1997-1999. One is based on 10522 CCD observations made at Rio de Janeiro, Brazil; the other one is based on 956 visual observations made at Santiago, Chile. Linear fits applied to both data sets give a standard deviation of $\pm 0^{\prime \prime} 365$ and $\pm 0^{\prime \prime} 360$ for each daily mean value of the solar radius for Rio de Janeiro and Santiago respectively. During the period of this analysis, the apparent solar radius had an annual variation of -0. . $060 \pm 0$.'013 according to the CCD observations of Rio de Janeiro and of +0. .' $213 \pm 0$. . 018 according to the visual observations of Santiago. We show here that this discrepancy could be due to systematic effects, probably of instrumental origin, that produce significant internal inconsistencies in the results of Rio de Janeiro. The drift of the apparent solar radius observed with the astrolabe of Santiago during 1997-1999 is consistent with former results obtained with this instrument since 1990. These results show that the apparent semidiameter of the Sun
\end{abstract} \\ varies in phase with solar activity (Noël 1997, 2001).
}

Key words. astrometry - Sun: activity - Sun: fundamental parameters

\section{Introduction}

According to prevalent theories of stellar physics one should expect that a star like the Sun should have a radius increase not larger than a few parts in $10^{11}$ per year. Such small variation would be undetectable on time scales of human interest (Sofia et al. 1983). However, some authors claim to have detected significant variations of the apparent solar radius (Gilliland 1981; Laclare 1983; Ulrich \& Bertello 1995; Noël 1997; Costa et al. 1999). If these variations are real, they would constitute a serious challenge to stellar structure theory (Spruit 1994). The suspicion that the solar radius might be variable is a real motivation for establishing programs of systematic surveys.

Several observing techniques have been used for ground based measurements of the apparent solar diameter. However, they are based on two quite different approaches. One consists of a direct measurement of the angle subtended by the solar diameter, that is, by two opposite borders of the Sun. In the other, the diameter is obtained by timing the transit of opposite borders through fiducial marks on the sky or in the telescope focal plane. Elsewhere, we have stressed the advantages of this second approach with respect to the first (Noël 1999, 2001). This approach can be applied with the Danjon astrolabe (Danjon 1960), which works following the method of equal altitudes (Débarbat \& Guinot 1970) and has been

\footnotetext{
* e-mail: fnoel@das.uchile.cl
}

a powerful instrument in wide field astrometry (Fricke 1972; Eichhorn 1974). The solar semidiameter is obtained with the astrolabe by timing the drift of the Sun's image through a small circle of altitude or almucantar (Laclare 1983; Noël 1999). The observations are visual; however, one can obtain impersonal results by using a CCD camera (Laclare 1991; Sinceac 1998).

The legitimacy of visual observations for measuring the solar radius has been disputed since personal bias is one of the main unknowns, and it is rather difficult to estimate which part of the limb profile the eye is sensitive to. Thus, the absolute value of the solar diameter obtained from visual observations can be strongly affected by personal bias. However, due to uncertainties in other solar parameters and in the theory of solar structure, solar models can be constructed that match a wide range of radius values. Therefore, for solar physics, eventual variations of the Sun's semidiameter are more interesting than its actual value (Ribes et al. 1991; Toulmonde 1995). Nevertheless, if the goal of a program of visual observations of the Sun is the research of radius variations, it must be stressed that the observations should be made by a single observer in order to avoid or at least to minimize personal effects.

More credibility can be given to observed changes in the solar diameter if the evidence comes from observations made at different sites, with different observing techniques and performed by independent research teams. Here we discuss two sets of measurements of the apparent solar radius. One was obtained from $\mathrm{CCD}$ observations made 
with the Danjon astrolabe of Observatório Nacional at Rio de Janeiro, Brazil. The other one is based on visual observations made by a unique observer with the Danjon astrolabe of the National Astronomical Observatory of Cerro Calán at Santiago, Chile. Since both data sets are rather dense and they cover the same period of observations, 1997-1999, and were obtained in quite independent research programs, they provide a good opportunity for comparing CCD and visual measurements of the solar radius.

\section{Solar observations at Rio de Janeiro and Santiago}

The program of CCD observations with the astrolabe of Observatório Nacional at Rio de Janeiro for monitoring variations of the apparent diameter of the Sun, has been described by Jilinski et al. $(1998,1999)$. Regular observations started in January, 1997, and they were in progress at least until May 2000. In this astrolabe the small circle of altitude is defined by means of a reflecting prism with a variable angle which permits observations at several zenith distances from about $27^{\circ}$ to $58^{\circ}$ (Jilinski et al. 1998). These zenith distances and the latitude of Rio de Janeiro $(-22.9)$ allow observations of the Sun all year round. Since a prism with a variable angle permits several observations of the Sun during the day, the data set of Rio de Janeiro is based on a very large number of solar radius measurements (10522), that were performed during the relatively short period $1997-1999$. The daily mean values analyzed in this paper were obtained in electronic form via Internet from the homepage of Observatório Nacional, Brazil. According to Jilinski et al. (1999) these results are available for public access; therefore, they should be considered as definitive and under this understanding they are used and discussed in this paper.

A detailed description of the project of solar astrometry with the modified Danjon astrolabe of the National Astronomical Observatory of Cerro Calán at Santiago was given by Chollet \& Noël (1993) and by Noël (1997, 1999, 2001). The observations at Santiago are made visually at two zenith distances, $30^{\circ}$ and $60^{\circ}$, defined by CERVIT reflecting prisms. Since the astrolabe latitude is $-33^{\circ} 4$, the Sun can be observed all year round at $60^{\circ}$ and from October 6th until March 7 th at $30^{\circ}$. At most four observations a day are possible; these are the east and west observations either at $30^{\circ}$ or at $60^{\circ}$ zenith distances. Therefore, the number of solar radius measurements, 956, during 1997-1999 is far less than the figure quoted above for Rio de Janeiro. Nevertheless, we consider that the data sets of Rio de Janeiro and Santiago are quite dense and they cover a sufficiently long interval of time to allow a valid comparison of both sets of results and to research eventual variations of the apparent semidiameter of the Sun.

\section{Results and discussion}

The daily results of the solar radius measurements made at Rio de Janeiro and Santiago during 1997-1999 are plotted in Fig. 1 as a function of time expressed in besselian years. Each point represents a mean value of the semidiameter computed with the individual measurements made during the same day and reduced to the astronomical unit. On average, the points of Rio de Janeiro are based on 13 CCD individual measurements. For Santiago each point is the mean value of only two daily visual measurements; one at the east transit of the Sun and the other one at the west transit, either at $30^{\circ}$ or $60^{\circ}$ zenith distance. For a clear comparison, a positive offset of one arc second has been applied to the results of Santiago.

A least squares linear fit applied to both sets of daily results gives standard deviations of \pm 0 ". 365 for the 755 results of Rio de Janeiro and $\pm 00^{\prime \prime} 360$ for the 478 results of Santiago. These figures do not show a higher precision of the CCD observations than that of the visual ones, as one should expect. Furthermore, it must be considered, as stated above, that the daily values of Rio de Janeiro plotted in Fig. 1 have already a much higher degree of smoothing than those of Santiago. Therefore, the dispersion of visual observations is, at least in this case, lower than that of the CCD observations. This could be due to better local conditions for astrometric work at Santiago than those existing at Rio de Janeiro. However, it could be also a confirmation that in certain circumstances, visual measurements of the solar radius can be more accurate than those made with photoelectric devices, as has been claimed by other authors (Wittmann 1997, 2000; Toulmonde 1995), and as we shall see below.

It is apparent in Fig. 1 that some results of Rio de Janeiro show large deviations with respect to the linear fit. The results at the end of 1999 look abnormal which may be due to some strong systematic effect. According to Jilinski (2000) the solar radius measurements with large deviations obtained with the CCD astrolabe at Rio de Janeiro have two main causes. These are errors in the CCD pixel scale and matrix orientation in the azimuth direction, and rather high levels of air humidity during the observations.

The first type of errors occur more frequently during those periods around the end of December, when according to the latitude of Rio de Janeiro, the Sun's hour angle has extreme values at the instant of the almucantar transit and there is no motion in azimuth of the solar image (Débarbat \& Guinot 1970). In such conditions and according to the reduction method applied at Rio de Janeiro, it is rather difficult to determine the correct pixel scale and matrix orientation in the azimuth direction (Jilinski 2000).

The effect of high levels of air humidity is more pronounced during June-July when, due to the latitude of Rio de Janeiro, the observations are made between $45^{\circ}$ and $55^{\circ}$ zenith distances. During the east or morning observations, the humidity is sometimes so high that it produces condensation in the instrument and the results are affected by 


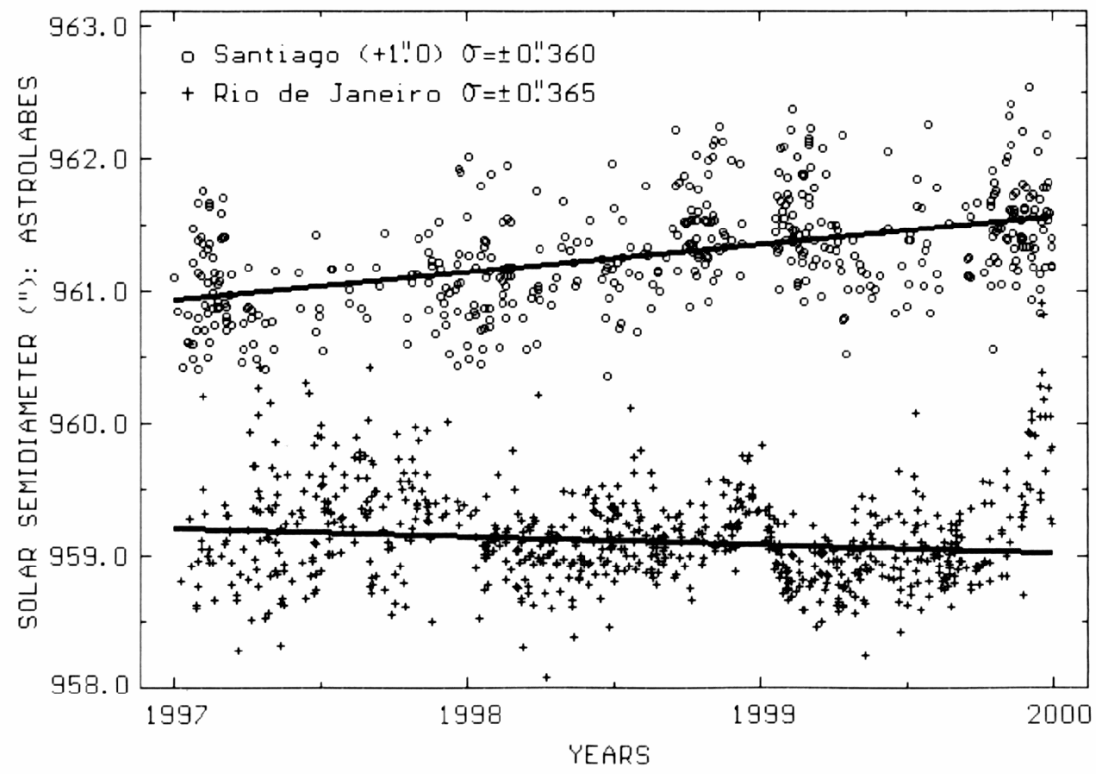

Fig. 1. Daily mean values of solar radius measurements made during 1997-1999 by means of CCD observations with the astrolabe of Rio de Janeiro $(+)$ and by means of visual observations with the astrolabe of Santiago (o). On average, each point for Rio de Janeiro is the mean of $13 \mathrm{CCD}$ measurements of the solar radius. For Santiago is the mean of 2 visual measurements. $\sigma$ is the standard deviation of the linear fits. For a more clear comparison of both data sets, the results of Santiago are incremented by one arc second. Some results of Rio de Janeiro with large deviations, like those close to the end of 1999, are due to systematic effects described in the text.

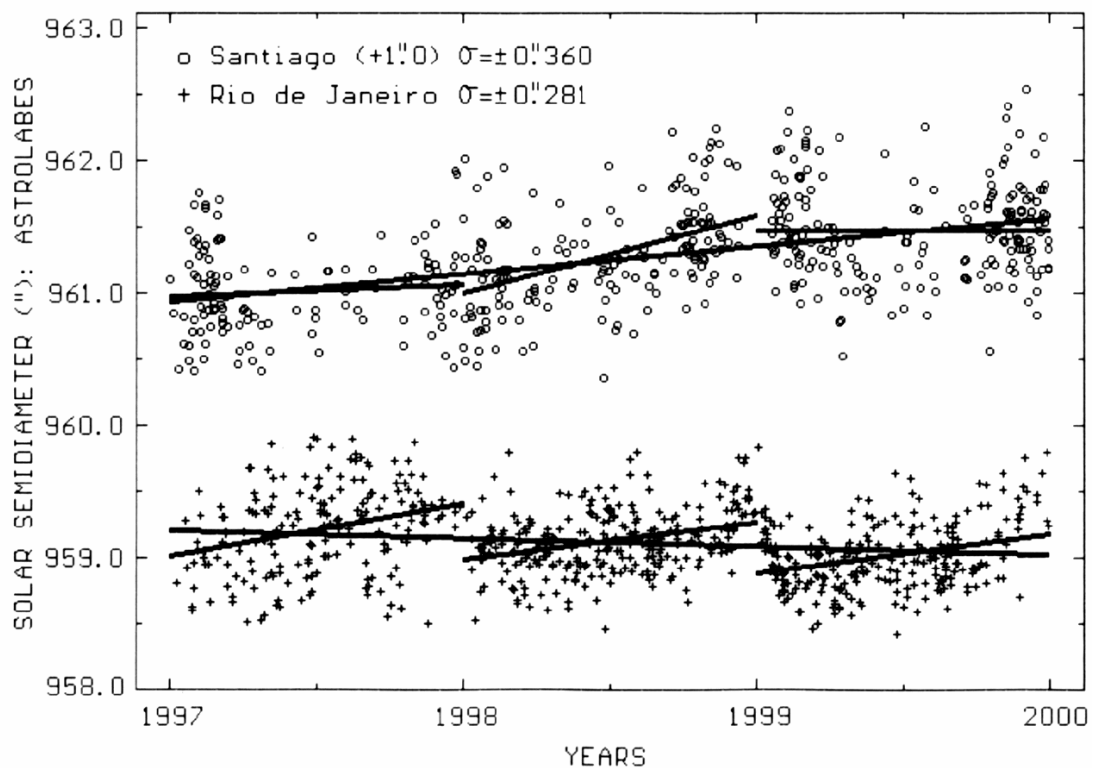

Fig. 2. Daily means of solar radius measurements obtained during 1997-1999 with the CCD astrolabe of Rio de Janeiro (+) and with the visual astrolabe of Santiago (o). The data for Santiago are the same as those of Fig. 1. For Rio de Janeiro we plotted only the measurements with residuals lower than $2 \sigma$ of Fig. 1 . Annual linear fits, applied to both set of results, show significant discontinuities in the radius measurements of Rio de Janeiro, due probably to changes introduced in the instrumental system (see text).

undetermined systematic errors. However, the tendency of both factors is to produce abnormally high values of the solar radius (Jilinski 2000).

Following this explanation, and in order to remove or at least to minimize the effect of the abnormal values, we have kept only the radius measurements of Rio de Janeiro with residuals lower than $2 \sigma$. Thus, 40 results were elimi- nated and the remaining 715 are plotted in Fig. 2 with the results of Santiago. A new linear fit now gives a standard deviation of \pm 0 ". 281 for the radius measurements of Rio de Janeiro.

With respect to variations in time of the solar radius during 1997-1999, and as can be seen in Figs. 1 and 2, the results from Santiago show a significant positive drift of 
Table 1. Parameters of linear fits applied to solar radius measurements by means of CCD observations with the astrolabe of Rio de Janeiro and by means of visual observations with the astrolabe of Santiago during 1997-1999. $A$ and $B$ are the coefficients in the equation of the least square fitted lines and $\sigma$ is the standard deviation of the linear fits. $n$ is the number of daily mean values of the solar semidiameter involved in the fits.

\begin{tabular}{|c|c|c|c|c|c|c|c|c|c|c|c|c|}
\hline \multirow[b]{2}{*}{ Year } & \multicolumn{6}{|c|}{ Rio de Janeiro } & \multicolumn{6}{|c|}{ Santiago } \\
\hline & \multicolumn{2}{|c|}{$A$} & \multicolumn{2}{|c|}{$B$} & $\sigma$ & $n$ & \multicolumn{2}{|c|}{$A$} & \multicolumn{2}{|c|}{$B$} & $\sigma$ & $n$ \\
\hline 1997: & $959^{\prime \prime} 002=$ & 0.054 & $0.0^{\prime \prime} 404=$ & 0.094 & $\pm 0^{\prime \prime} 319$ & 195 & $959 . \prime 964=$ & $0^{\prime \prime} 048$ & $0^{\prime \prime} 096=$ & 0.091 & $\pm 0 . \prime 333$ & 120 \\
\hline 1998: & 958.982 & 0.029 & 0.282 & 0.051 & 0.228 & 270 & 959.988 & 0.051 & 0.589 & 0.089 & 0.347 & 158 \\
\hline 1999: & 958.875 & 0.030 & 0.304 & 0.058 & 0.245 & 250 & 960.471 & 0.049 & -0.006 & 0.076 & 0.365 & 200 \\
\hline $97-99:$ & 959.199 & 0.023 & -0.060 & 0.013 & 0.281 & 715 & 959.933 & 0.034 & 0.213 & 0.018 & 0.360 & 478 \\
\hline
\end{tabular}

the apparent radius, while those of Rio de Janeiro have a negative drift. However, both figures give the impression that there are apparent discontinuities in the results of Rio de Janeiro around 1998.0 and 1999.0. This impression is confirmed by annual linear fits applied to the results of Rio de Janeiro and Santiago given in Fig. 2. The parameters of the annual and tri-ennial fits plotted in Fig. 2 are given in Table 1. From these parameters we have that the annual radius variation for Santiago, according to the average of the annual and tri-ennial fits, is

$+0.226 \pm 0$. 049 and $+0^{\prime \prime} .213 \pm 0 . .018$

respectively. There is no significant difference between both values, as one should expect from a self-consistent data set. However, the results of Rio de Janeiro do not show such internal consistency, since in this case the corresponding values are

$+0.330 \pm 0$ ". 041 and $-00^{\prime \prime} 060 \pm 0 . .013$.

Therefore, during each year, the observed solar diameter increases; however, the total variation during the whole period is negative. This significant and striking discrepancy deserves an explanation. Some hints for explaining such a discrepancy can be found in Jilinski et al. (1999) where the observations of Rio de Janeiro between 1997.0 and 1998.5 are discussed. According to these authors (ibid., p. 227),

"The period of observations (at Rio de Janeiro) can be divided in two, before and after January 1st, 1998, when the filter after the image vehicle was swapped by one with a narrower bandpass (italics are mine)... With the new filter the width of the measured borders of the Sun became narrower and as such the definition of the limb of the Sun became more precise and the error of the diameter measurements decrease a little."

The authors claim that the new filter with a narrower bandpass produced only a diminution of the large scatter of the initial results since later on they say (ibid., p. 228),

"The large scatter of the initial observations is evident, although no systematic offset is apparent (italics are mine). On the contrary, the average values for the 1997 and 1998 summer observations agree well."

We agree that the 1997 results of Rio de Janeiro show a larger scatter than those of 1998 and 1999, this is clearly shown by the values given in Table 1 . However, we cannot agree with the assertion of the authors, that there is no systematic offset around 1998.0. On the contrary, according to Fig. 2 and Table 1, there is a strong discontinuity in the results of Rio de Janeiro precisely around that epoch. Furthermore, such discontinuity can be seen also in Fig. 2 of the paper of Jilinski et al. (1999). The values of Table 1 give an offset of -0 '! $424 \pm 0$ "' 112 in the solar radius observed at Rio de Janeiro around 1998.0. Following the assertion of Jilinski et al. (1999) quoted above, we presume that this offset is of instrumental origin and it might be associated with the installation of the new filter mentioned by the authors.

Concerning the second discontinuity in the results of Rio de Janeiro around 1999.0, it amounts to $-00^{\prime \prime} 389 \pm$ 0 .'066 according to the values of Table 1 . However, the epoch 1999.0 is not included in the observational period discussed by Jilinski et al. (1999). Therefore, there are no hints about its probable origin. Nevertheless, we presume that it could also be of instrumental origin.

With respect to the visual results of Santiago, the annual linear fits in Fig. 2 show also discontinuities in 1998.0 and 1999.0. However, from the values of Table 1, the offsets for Santiago at 1998.0 and 1999.0 are $-0 . \prime 072 \pm 0$ '. 115 and -0 "' $107 \pm 0$ ". 114 respectively. Both values are not significant and they can be explained by cumulative effects of observational accidental errors.

According to Table 1 the linear fit 97-99 gives discrepant values of the annual drift of the solar radius for Rio de Janeiro and Santiago. The CCD results of Rio de Janeiro show a decrease in the apparent radius of $-0{ }^{\prime \prime} 060 \pm 0$ !'013 per year, while the visual results of Santiago show an annual increase of $+0^{\prime \prime} 213 \pm 00^{\prime \prime} 018$. However, taking the average of parameter $B$ of the annual linear fits given in Table 1, the discrepancy disappears, since in this case the yearly variation of the solar radius is $+00^{\prime \prime} 330 \pm 0$ ! 041 and $+00^{\prime \prime} 226 \pm 0$ !'049 for 
Rio de Janeiro and Santiago respectively. Both values are fairly consistent.

The annual variation of the apparent solar radius shown by the visual astrolabe of Santiago during 19971999 is in agreement with other results obtained with this instrument since 1990. According to these results, the observed solar radius varies in phase with the variation in sunspot number. The first report was given by Noël (1997) and since then, and as can be seen in Fig. 3, the solar cycle dependence of the apparent radius has been amply confirmed by our later results (Noël 1999, 2000, 2001). In Fig. 3 are plotted the annual means of sunspot number provided by the Sunspot Index Data Center (SIDC) of Bruxelles, Belgium, and the annual deviations from the mean of the apparent solar radius observed at Santiago. We also have plotted the results obtained by Ulrich \& Bertello (1995) with the solar magnetograph of the Mount Wilson Observatory. The radius measurements at Mount Wilson are a by-product of the daily full-disk magnetograms. The radius is obtained by means of photoelectric scans of the solar image using the $525.0 \mathrm{~nm}$ radiation of neutral iron. The complete time series of Mount Wilson results covers the period 1982-1994 and also show a significant variation of the apparent radius in phase with solar magnetic activity (Ulrich \& Bertello 1995).

Concerning the results plotted in Fig. 3, it should be considered that there are other modern results, based on different observing techniques, that also show positive correlations between time variations of the apparent radius of the Sun and solar activity. For instance, an analysis made by Basu (1998) of homogenised data sets of solar radius measurements covering the last three centuries gives a statistically significant relationship between larger diameters of the Sun and higher levels of solar activity. Radio-observations of the Sun at $48 \mathrm{GHz}$ made during the period 1991-1993 at Itapetinga Radio Observatory, São Paulo, Brazil, show a decrease of the measured radius as the solar cycle declines (Costa et al. 1999). In a 3 years experiment (1996-1998) to detect solar diameter fluctuations with the Michelson Doppler Imager (MDI) on board the Solar and Heliospheric Observatory (SOHO), it was found that the radius of the Sun increases approximately in a linear way with the number of sunspots (Emilio et al. 2000, see also Gough 2001).

\section{Conclusions}

According to some authors, the CCD measurements of the solar radius with astrolabes make possible a practical definition of the solar border through the limb darkening function (Laclare et al. 1999). Concerning visual measurements, the CCD results have a great advantage since they should be free from personal bias. On the other hand, they allow a measure of the image quality expressed by the seeing parameter of Fried (1966) which can be related to errors affecting the solar radius measurements (Irbah et al. 1994).

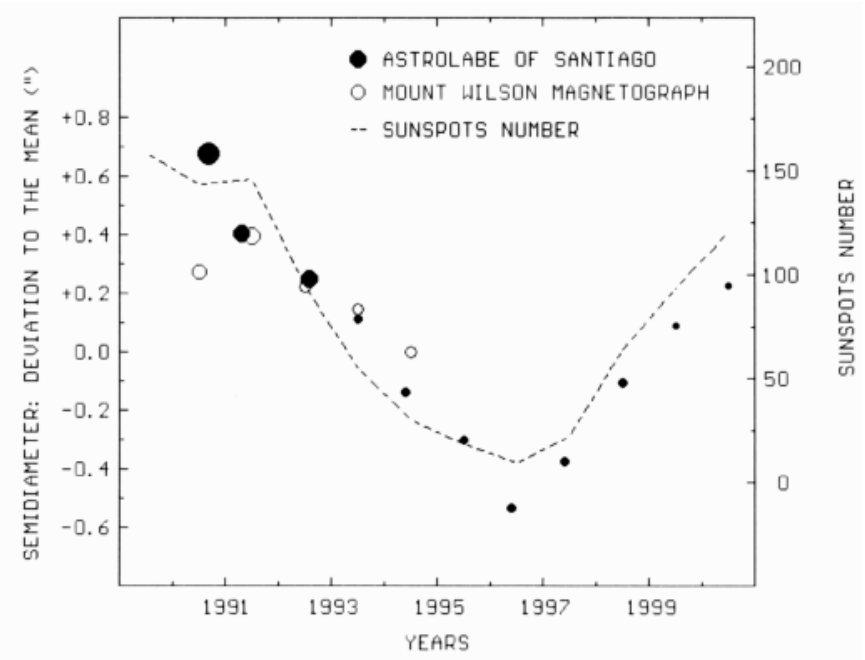

Fig. 3. Annual mean values of deviations from the mean of solar radius measurements made with the visual astrolabe of Santiago (filled circles) and annual mean values of sunspot number. The open circles are similar results to those of Santiago, obtained with the solar magnetograph of the Mount Wilson Observatory using the $525 \mathrm{~nm}$ spectral line of neutral iron. The size of the circles for Santiago and Mount Wilson gives the order of the mean error of the annual values.

However, and at least for the time being, this advantages could have only a formal character, since as we have shown in this paper, in practice, CCD measurements of the solar diameter with an astrolabe can be strongly affected by systematic effects that produce quite significant distorsion of the results. We have seen also that apparently, no such large systematic effects are evident in visual observations when they are performed by a single experienced observer. On the other hand, and according to Fig. 1, the original CCD results of Rio de Janeiro in spite of its higher degree of smoothing (see Sect. 3), show a dispersion almost equivalent to that of the visual results of Santiago. The mean daily values of the solar radius in Fig. 1 are based on the average on 13 CCD measurements at Rio de Janeiro and on a fixed number of only two visual measurements at Santiago. Therefore, to obtain a precision equivalent to one visual observation at Santiago, one needs 6 CCD observations at Rio de Janeiro. It is interesting to note that a somewhat similar equivalence has been found between visual and CCD solar radius measurements, but performed with an instrument different to the Danjon astrolabe. Using the Gregory-Coudé Telescope of Observatorio del Teide, Tenerife, Wittmann $(1997,1998)$ claims that 8 CCD drift scan measurements of the solar radius are required in order to get the accuracy of one visual drift timing. According to this author, the reason is the fluctuations of the limb position of the Sun and image blurring due to the atmosphere. These effects can be monitored with the eye but not with a detector. Moreover, besides the systematic effects of the CCD observations that we have seen above, Wittmann \& Bianda (2000) observed that the value of the solar radius obtained from CCD 
measurements is somewhat dependent on the threshold of the camera, which in turn dependes on gain, air transparency, etc.

Finally, we note that the pioneer group in the application of a CCD camera to the Danjon astrolabe to measure the solar radius recognizes that, contrary to what one would expect, the CCD observations do not give significantly better results than those obtained from visual observations (Laclare et al. 1999). Following what we have seen in this paper, we think that this assertion is somewhat conservative.

Acknowledgements. The project of the Danjon astrolabe at Santiago is a joint research program in astrometry between the European Southern Observatory (ESO) and Universidad de Chile. The solar radius data of Rio de Janeiro used in this paper have been produced at the Observatório Nacional (CNPq, Brazil). I wish to thank the referee of this paper, Dr. Axel Wittmann, for a very interesting interchange of ideas.

\section{References}

Basu, D. 1998, Solar Phys., 183, 291

Chollet, F., \& Noël, F. 1993, A\&A, 276, 655

Costa, J. E. R., Silva, A. V. R., Makhmutov, V. S., et al. 1999, ApJ, 520, L63

Danjon, A. 1960, in Telescopes, ed. G. P. Kuiper, \& B. M. Middlehurst (The University of Chicago Press), 115

Débarbat, S., \& Guinot, B. 1970, La méthode des hauteurs égales en astronomie (Gordon and Breach Eds., Paris)

Eichhorn, H. 1974, Astronomy of Star Positions (Frederick Ungar Publishing Co., New York), 49

Emilio, M, Kuhn, J. R., Bush, R. I., \& Scherrer, P. H. 2000, ApJ, 543, 1007

Fricke, W. 1972, ARA\&A, 10, 107
Fried, D. L. 1966, J. Opt. Soc. Am., 56, 1372

Gilliland, R. L. 1981, ApJ, 248, 1114

Gough, D. 2001, Nature, 410, 313

Irbah, A., Laclare, F., Borgnino, J., \& Merlin, G. 1994, Solar Phys., 149, 213

Jilinski, E. G., Puliaev, S., Penna, J. L., et al. 1998, A\&AS, 130,317

Jilinski, E. G., Puliaev, S., Penna, J. L., Andrei, A. H., \& Laclare, F. 1999, A\&AS, 135, 227

Jilinski, E. G. 2000, private communication

Laclare, F. 1983, A\&A, 125, 200

Laclare, F. 1991, C. R. Acad. Sci. Paris, Ser. II, 313, 323

Laclare, F., Delmas, C., Sinceac, V., \& Chollet, F. 1999, C. R. Acad. Sci. Paris 327, Ser. II b, 645

Noël, F. 1997, A\&A, 325, 825

Noël, F. 1999, A\&A, 343, 1001

Noël, F. 2001, in The Shape of the Sun, V Summer School on Solar Astrophysics, Saint Pierre d'Oleron, France, ed. J. P. Rozelot, Lectures Notes in Astrophysics (Springer-Verlag), in press

Ribes, E., Beardsley, B., Brown, T. M., et al. 1991, in The Sun in Time, ed. C. P. Sonett, M. S. Giampapa, \& M. S. Matthews (The University of Arizona Press), 59

Sinceac, V. 1998, Ph.D. Thesis, Observatoire de Paris, France

Sofia, S., Dunham, D. W., Dunham, J. B., \& Fiala, A. D. 1983, Nature, 504, 322

Spruit, H. C. 1994, in The Solar Engine and its Influence on Terrestrial Climate, ed. E. Nesme-Ribes, NATO ASI Ser., V125 (Springer-Verlag, Berlin Heidelberg), 107

Toulmonde, M. 1995, Ph.D. Thesis, Observatoire de Paris, France

Ulrich, R. K., \& Bertello, L. 1995, Nature, 377, 214

Wittmann, A. D. 1997, Solar Phys., 171, 231

Wittmann, A. D. 1998, private communication

Wittmann, A. D., \& Bianda, M. 2000, in Proc. 1st Solar \& Space Weather Euroconference, The Solar Cycle and Tesrrestrial Climate (ESA SP-463, December 2000), 113 